\title{
Evaluation of the SpeeDx Carba (beta) multiplex real-time PCR assay for detection of NDM, KPC, OXA-48-like, IMP-4-like and VIM carbapenemase genes
}

Amanda Bordin ${ }^{1 \dagger}$, Ella Trembizki ${ }^{1 \dagger}$, Madeline Windsor ${ }^{2}$, Rachel Wee², Lit Yeen Tan², Cameron Buckley ${ }^{1}$, Melanie Syrmis ${ }^{1,3}$, Haakon Bergh ${ }^{3}$, Kyra Cottrell ${ }^{1}$, Hosam M. Zowawi ${ }^{1,4,5,6,7}$, Hanna E. Sidjabat ${ }^{1}$, Patrick N. A. Harris ${ }^{1,3}$, Graeme R. Nimmo ${ }^{3,8}$, David L. Paterson ${ }^{1}$ and David M. Whiley ${ }^{1,3^{*}}$

\begin{abstract}
Background: Carbapenemase-producing organisms (CPOs) have emerged as antibiotic-resistant bacteria of global concern. Here we assessed the performance of the Carba (beta) assay, a multiplex real-time PCR assay developed by SpeeDx for the detection of key carbapenemase-encoding genes: KPC, NDM, OXA-48-like, IMP-4-like, and VIM.

Methods: DNA extracts of 180 isolates were tested with the Carba (beta) assay, using previously validated in-house TaqMan probe assays for the relevant carbapenemase genes as the reference standard. The Carba (beta) assay was then directly used to screen 460 DNA extracts of faecal specimens, with positive results subjected to the aforementioned in-house assays plus Sanger sequencing.
\end{abstract}

Results: The Carba (beta) assay correctly identified the presence of the respective carbapenemase genes in 154 of 156 isolates and provided negative results for all 24 non-CPO isolates. Two isolates provided positive results for OXA-48-like carbapenemase by the Carba (beta) assay only. The Carba (beta) assay had sensitivities of 100\% for all targets, and specificities of 100\% for KPC, NDM, IMP-4-like, and VIM targets, and 98.5\% for OXA-48-like targets. When applied directly to faecal specimens, eight samples were positive by the Carba (beta) assay, two of which were confirmed by in-house TaqMan probe PCR or DNA sequencing.

Conclusions: The Carba (beta) assay is highly sensitive and specific for detecting key carbapenemase genes in isolates. Further testing is required to assess this assay's suitability for direct screening of clinical specimens.

Keywords: Multiplex, Real-time, PCR, Carbapenemase, KPC, NDM, OXA-48, IMP-4, VIM

\section{Background}

Carbapenemase-producing organisms (CPOs) are antibioticresistant bacteria of global concern. The United States Centers for Disease Control and Prevention have assigned an "urgent" threat level to carbapenemresistant Enterobacteriaceae [1], and the World Health Organisation has identified carbapenemase-producing

\footnotetext{
*Correspondence: d.whiley@uq.edu.au

${ }^{\dagger}$ Amanda Bordin and Ella Trembizki contributed equally to this work.

${ }^{1}$ The University of Queensland, UQ Centre for Clinical Research, Brisbane, Queensland, Australia

${ }^{3}$ Pathology Queensland Central Laboratory, Brisbane, Queensland, Australia Full list of author information is available at the end of the article
}

Klebsiella pneumoniae and Escherichia coli as "bacteria of international concern" [2]. Infections caused by CPOs are, at best, difficult-to-treat, and have sparked concerns over the potential of untreatable infections causing significant morbidity and mortality in health care settings worldwide [2-5]. Rapid detection of CPO infection and subsequent implementation of infection control procedures is pivotal to manage infection and to prevent further nosocomial transmission [6]. Carbapenemase genes of particular clinical importance globally include Klebsiella pneumoniae carbapenemase (KPC), New Delhi metallo- $\beta$-lactamase (NDM), oxacillin-hydrolyzing48-like (OXA-48-like), imipenemase-4-like (IMP-4-like), 
and Verona integron-encoded metallo- $\beta$-lactamase (VIM) genes $[3,5,7]$. In this study, we assessed the performance of the new SpeeDx Carba (beta) multiplex real-time PCR assay for detection of these carbapenemase genes using a panel of $\mathrm{CPO}$ isolates. As direct screening of patient samples (particularly rectal swab and faecal samples for intestinal carriage) is beginning to find its way into routine practice to inform infection control $[8,9]$, we then also applied the method to screen for carbapenemase genes directly in faecal specimens from local patients.

\section{Methods Isolates}

The sensitivity and specificity of the Carba (beta) assay for carbapenemase detection was assessed by testing an isolate panel of CPOs $(n=156)$ [10-14] and non-CPOs $(n=24)$ of various bacterial species, and comparing the results to in-house TaqMan probe real-time PCR methods. By origin, CPO isolates were from Pakistan $(n=51)$, Saudi Arabia $(n=42)$, Australia $(n=19)$, Turkey $(n=17)$, USA $(n=17)$, Bahrain $(n=5)$ and Qatar $(n=5)$, and the 24 non-CPO isolates were from Australia. Isolate extracts were prepared using a simple boil-up method whereby single colonies were suspended in $1.0 \mathrm{~mL}$ of sterile water to provide a concentration consistent with a $0.5 \mathrm{McF}$ arland standard, before being heated at $95^{\circ} \mathrm{C}$ for $30 \mathrm{~min}$.

\section{Faecal specimens}

Remnant DNA extracts from faecal samples $(n=460)$ submitted for routine gastrointestinal testing at Pathology Queensland were tested. In brief, the faecal samples were collected from November 2016 to January 2017 and were extracted at Pathology Queensland using the MagNA Pure 96 System (Roche, Australia) according to manufacturer's instructions. All samples were tested with the Carba (beta) assay. Samples providing positive results in the Carba (beta) assay were subjected to in-house TaqMan probe PCR assays and Sanger sequencing.

\section{SpeeDx Carba (beta) PCR}

The Carba (beta) assay (SpeeDx Pty Ltd., Australia) was designed to detect KPC, NDM, OXA-48-like, IMP-4-like, and VIM carbapenemase-encoding genes in a single multiplex reaction. The assay utilises PlexZyme (formerly known as MNAzyme) real-time detection technology [15-17] which has advantages in maintaining sensitivity and specificity in multiplex. Detection is predicted for all current known variants of VIM and KPC; NDM-1-7; OXA-48, 162, 163, 181, 204, 232, 244, 245, 247, 370; and IMP-1, 2, 4, 6, 8, 10, 13, 18, 19, 20, 24, 26, 27, 30, 33, 37, $38,42,48,49,52$. The Carba (beta) PCR reaction was prepared as per the manufacturer's instructions, with $15.0 \mu \mathrm{L}$ of master mix and $5.0 \mu \mathrm{L}$ of isolate suspension or faecal extract. Reactions were amplified on the ABI7500 realtime PCR instrument (ThermoFisher Scientific, Australia) using the manufacturer's cycling conditions: an initial $95^{\circ} \mathrm{C} 2 \mathrm{~min}$ hold, followed by 10 touch-down cycles at $95^{\circ} \mathrm{C}$ for $5 \mathrm{~s}$ and $61{ }^{\circ} \mathrm{C}\left(-0.5^{\circ} \mathrm{C}\right.$ per cycle $)$ for $30 \mathrm{~s}$, followed by 40 cycles at $95^{\circ} \mathrm{C}$ for $5 \mathrm{~s}$ and $52^{\circ} \mathrm{C}$ for $40 \mathrm{~s}$. KPC was reported by FAM, NDM by JOE, OXA-48-like by Texas Red, IMP-4-like and VIM by Cy5 (and therefore IMP-4-like and VIM results could not be distinguished), and the internal control by TAMRA. The internal control DNA was diluted to provide an expected cycle threshold (Ct) value of approximately 22 cycles as per the manufacturer's instructions.

\section{In-house PCR}

In-house PCR methods utilised previously described primers and probes (see Table 1) for KPC [18], NDM [18], OXA-48-like [19], IMP-4-like [20], VIM [20] and pan-bacterial 16S rRNA [18] (the latter serving as an internal DNA extraction and amplification control). NDM, KPC and 16S rRNA reactions were performed together in a triplex, IMP-4-like and VIM as a duplex, and OXA-48-like as a singleplex reaction. The reverse primer of the OXA-48-like reaction was modified from the original [19] ( $G$ to $C$ at position 15) based on sequence data from GenBank. The QuantiFast Probe

Table 1 Oligonucleotides for in-house assays in this study

\begin{tabular}{|c|c|c|c|c|}
\hline Set & Forward primer $\left(5^{\prime}-3^{\prime}\right)$ & Reverse primer $\left(5^{\prime}-3^{\prime}\right)$ & Probe & Reference \\
\hline $\mathrm{KPC}^{\mathrm{a}}$ & GGCCGCCGTGCAATAC & GCCGCCCAACTCCTTCA & FAM-TGATAACGCCGCCGCCAATTTGT-BHQ1 & [18] \\
\hline $\mathrm{NDM}^{\mathrm{a}}$ & GACCGCCCAGATCCTCAA & CGCGACCGGCAGGTT & VIC-TGGATCAAGCAGGAGAT-MGB-NFQ & [18] \\
\hline OXA-48-like & GTAGCAAAGGAATGGCAA & CCTTGCTGCTTATTCTCA ${ }^{c}$ & FAM-TCC $(+A) G A(+G) C A(+C) A A(+C) T A C G-D a b c y l$ & [19] \\
\hline IMP-4-like ${ }^{b}$ & GGCAGTATTTCCTCTCATTT & GCAGCTCATTAGTTAATTCAG & FAM-CATAGTGACAGCACGGGCGGAAT-BHQ1 & [20] \\
\hline$V I M^{b}$ & CGCGGAGATTGAGAAGCAAA & AGCCGCCCGAAGGACATC & HEX-TTGGACTTCCTGTAACGCGTGCA-BHQ1 & [20] \\
\hline $16 \mathrm{~S} r \mathrm{RNA} \mathrm{A}^{\mathrm{a}}$ & TGGAGCATGTGGTTTAATTCGA & TGCGGGACTTAACCCAACA & Quasar670-CACGAGCTGACGACARCCATGCA-BHQ2 & [18] \\
\hline
\end{tabular}

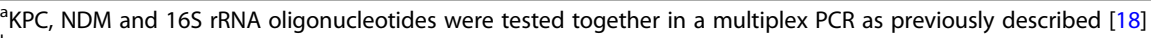

${ }^{\mathrm{b}} \mathrm{IMP}-4$-like and VIM oligonucleotides were tested together in a multiplex PCR as previously described [20]

'The lower case base differed to that of the original primer ( $C$ instead of $G$ ) and was substituted based on OXA-48-like sequence data on the Genbank database

' + ' indicates locked nucleic acid (LNA) bases; MGB minor groove binder, BHQ black hole quencher, NFQ non-fluorescent quencher 
PCR Kit (QIAGEN, Australia) was used as the basis for the reaction mixes. Each mix comprised $0.5 \mu \mathrm{M}$ of forward and reverse primers, $0.25 \mu \mathrm{M}$ of probe and $2.0 \mu \mathrm{L}$ of isolate preparation or faecal extract in a total reaction volume of $20.0 \mu \mathrm{L}$. Reactions were amplified on the Rotor-Gene (QIAGEN, Australia) real-time PCR instrument using an initial $95^{\circ} \mathrm{C}$ hold for 3 min followed by 45 cycles at $95^{\circ} \mathrm{C}$ for $3 \mathrm{~s}$ and $60^{\circ} \mathrm{C}$ for $30 \mathrm{~s}$.

\section{PCR controls and environmental practices}

All PCR test runs included relevant positive control reaction/s to assess PCR amplification and at least two non-template (sterile $\mathrm{H}_{2} \mathrm{O}$ ) negative control reactions to check for contamination. Sample extraction, mix preparation, DNA preparation/handling, and PCR amplification were all conducted in separate areas using dedicated equipment.

\section{Detection limit comparisons}

To assess the detection limits of the Carba (beta) assay in comparison to the in-house assays, five $\mathrm{CPO}$ isolates were selected as representatives of each carbapenemase gene. DNA extracts of each of these samples were serially diluted ten-fold and run in three to five replicates (see Table 5) through the Carba (beta) assay and the relevant in-house assay. Concentrations were based on the initial isolate extracted at $0.5 \mathrm{McFarland}$ standards (i.e. approximately $1.5 \times 10^{5}$ Colony Forming Units (CFUs) per $\mu \mathrm{L}$ of DNA extract). The detection limit for each target and assay was the lowest dilution where all replicates provided consistent positive $\mathrm{Ct}$ values.

\section{DNA sanger sequencing}

Select samples were subjected to DNA sequencing using the primers described in Table 2. Amplification was carried out in $20.0 \mu \mathrm{L}$ reaction volumes with $0.5 \mu \mathrm{M}$ of each primer and $2.0 \mu \mathrm{L}$ of isolate preparation or faecal extract using the QuantiTect SYBR PCR Kit (QIAGEN, Australia) as the basis for the reaction mix. Reactions were cycled on the Rotor-Gene instrument using an initial $95^{\circ} \mathrm{C}$ hold at $15 \mathrm{~min}$, followed by 45 cycles of $95^{\circ} \mathrm{C}$ for $30 \mathrm{~s}, 50^{\circ} \mathrm{C}$ for $30 \mathrm{~s}$ and $72^{\circ} \mathrm{C}$ for $60 \mathrm{~s}$, and finally a melt step from 45 to $95^{\circ} \mathrm{C}$ at $+0.2{ }^{\circ} \mathrm{C} /$ second. PCR products were submitted to the Australian Genomic Research Facility (AGRF; The University of Queensland) for Sanger sequencing and aligned to representative sequences from GenBank.

\section{Results}

Isolate extracts

The results for isolate extracts are summarised in Table 3.

Results from the Carba (beta) assay for 178 of 180 isolates (98.9\%) were in concordance with the reference in-house PCR methods. Of these 178 concordant isolates, 154 isolates were correctly-characterised CPOs (16 KPC, 50 NDM, 40 OXA-48-like, 21 VIM, 19 IMP-4-like, six isolates with NDM and OXA-48-like, one isolate with KPC and NDM, and one isolate with OXA-48-like and VIM) and 24 isolates were negative by both the Carba (beta) assay and in-house PCR. A further two isolates provided discrepant results; these two isolates (one Pseudocitrobacter faecalis and one Pseudomonas aeruginosa) provided concordant results for NDM and IMP-4-like/VIM targets respectively, but were positive for OXA-48-like by the Carba (beta) assay only. Using the in-house assay results as the reference standard, the Carba (beta) assay's sensitivities and specificities were both $100 \%$ for KPC, NDM, IMP-4-like and VIM genes, while for OXA-48-like genes sensitivity was $100 \%$ and specificity was $98.5 \%$.

An assessment of the $\mathrm{Ct}$ values for samples providing concordant positive results $(n=154)$ showed that 152 provided Carba (beta) assay Ct values of less than 20 cycles (7.0-18.9; mean 13.8 cycles). Two isolates (both $K$. pneumoniae and both positive for two genes) provided Carba (beta) assay Ct values of 12.6 cycles (KPC) and 34.7 cycles (NDM) for one isolate and 10.3 cycles (OXA-48-like) and 24.4 cycles (IMP-4-like/VIM) for the second isolate, with in-house PCR assay Ct values of 22.9 cycles (KPC) and 39.5 cycles (NDM), and 19.3 cycles (OXA-48-like) and 36.4 (VIM) respectively. The two isolates providing discrepant results ( $P$. faecalis and one $P$. aeruginosa, again both positive for two genes) both provided $\mathrm{Ct}$ values $<20$ cycles in the Carba (beta) assay for the concordant results (NDM and IMP-4like/VIM targets respectively), but Ct values of 27.8 or 30.9 cycles for OXA-48-like. These $\mathrm{Ct}$ values were the highest observed for OXA-48-like in the Carba (beta) assay.

All four isolates providing Carba (beta) assay Ct values greater than 20 cycles were re-extracted and retested. For each of these isolates, the target originally providing $\mathrm{Ct}$ values greater than 20 cycles was negative upon repeat, whereas the results associated with $\mathrm{Ct}$ values $<20$ cycles were reproducible.

It should be noted that the Carba (beta) assay cycling conditions utilise an initial 10-cycle touchdown step,

Table 2 Amplification and sequencing primers for Sanger sequencing

\begin{tabular}{llll}
\hline Set & Forward primer (5'-3') & Reverse primer (5'-3') & Amplicon size (bp) \\
\hline OXA-48-like & CCTCGATTTGGCGTGGTTA & TCCGATGTGGGCATATCCAT & 475 \\
IMP-4-like & GAAGGTGTTTATGTTCATACTTCGT & TCACTGTGACTTGGAACAACCAGTTTGC & 557 \\
VIM & GAGTTGCTITTGATTGATACAGC & TCGGTCGAATGCGCAGCACC & 291 \\
\hline
\end{tabular}


Table 3 Cycle threshold (Ct) results for isolate extract sample bank $(n=180)$. Results are grouped by carbapenemase type as determined by the Carba (beta) assay, then by species. Results highlighted in grey were negative upon repeated extraction and testing (discussed further in-text)

\begin{tabular}{|c|c|c|c|c|c|c|c|c|c|c|c|c|c|}
\hline \multirow{2}{*}{$\begin{array}{l}\text { Carbape- } \\
\text { nemase }\end{array}$} & \multirow[b]{2}{*}{ Organism } & \multirow[b]{2}{*}{$\mathrm{n}$} & \multicolumn{5}{|c|}{ Carba (beta) assay Ct values (range; mean) } & \multicolumn{6}{|c|}{ In-house assays Ct values (range; mean) } \\
\hline & & & KPC & NDM & $\begin{array}{l}\text { OXA-48- } \\
\text { like }\end{array}$ & $\begin{array}{l}\text { IMP-4- } \\
\text { like/VIM }\end{array}$ & IC & KPC & NDM & \begin{tabular}{|l|}
$\begin{array}{l}\text { OXA-48- } \\
\text { like }\end{array}$ \\
\end{tabular} & IMP-4-like & VIM & 16S rRNA \\
\hline \multirow{3}{*}{ KPC } & Enterobacter aerogenes & 1 & \begin{tabular}{|l|} 
POSITIVE \\
$(8.9)$
\end{tabular} & neg & neg & neg & $\begin{array}{l}\text { POSITIVE } \\
(20.0)\end{array}$ & $\begin{array}{l}\text { POSITIVE } \\
(20.4)\end{array}$ & neg & neg & neg & neg & \begin{tabular}{|l} 
POSITIVE \\
$(19.2)$
\end{tabular} \\
\hline & Escherichia coli & 14 & $\begin{array}{l}\text { POSITIVE } \\
(8.5-13.4 ; \\
11.6) \\
\end{array}$ & neg & neg & neg & $\begin{array}{l}\text { POSITIVE } \\
(22.0-22.8 ; \\
22.3)\end{array}$ & $\begin{array}{l}\text { POSITIVE } \\
(19.1-24.3 ; \\
22.0)\end{array}$ & neg & neg & neg & neg & \begin{tabular}{|l} 
POSITIVE \\
$(18.8-23.7$ \\
$22.3)$ \\
\end{tabular} \\
\hline & Klebsiella pneumoniae & 1 & $\begin{array}{l}\text { POSITIVE } \\
(12.4)\end{array}$ & neg & neg & neg & $\begin{array}{l}\text { POSITIVE } \\
(22.9)\end{array}$ & $\begin{array}{l}\text { POSITIVE } \\
(22.4)\end{array}$ & neg & neg & neg & neg & \begin{tabular}{|l} 
POSITIVE \\
$(22.0)$
\end{tabular} \\
\hline \multirow{10}{*}{ NDM } & Aeromonas hydrophila & 1 & neg & $\begin{array}{l}\text { POSITIVE } \\
(10.2)\end{array}$ & neg & neg & $\begin{array}{l}\text { POSITIVE } \\
(21.5)\end{array}$ & neg & \begin{tabular}{|l|} 
POSITIVE \\
$(21.3)$
\end{tabular} & neg & neg & neg & \begin{tabular}{|l|} 
POSITIVE \\
$(17.3)$ \\
\end{tabular} \\
\hline & Acinetobacter $s p$ & 1 & neg & $\begin{array}{l}\text { POSITIVE } \\
(14.5)\end{array}$ & neg & neg & $\begin{array}{l}\text { POSITIVE } \\
(21.9)\end{array}$ & neg & $\begin{array}{l}\text { POSITIVE } \\
(24.3)\end{array}$ & neg & neg & neg & \begin{tabular}{|l} 
POSITIVE \\
$(22.3)$
\end{tabular} \\
\hline & Citrobacter freundii & 3 & neg & $\begin{array}{l}\text { POSITIVE } \\
(7.0-12.5 ; \\
10.6)\end{array}$ & neg & neg & $\begin{array}{l}\text { POSITIVE } \\
(21.4-22.8 ; \\
22.0)\end{array}$ & neg & $\begin{array}{l}\text { POSITIVE } \\
(18.3-24.1 ; \\
22.0)\end{array}$ & neg & neg & neg & $\begin{array}{l}\text { POSITIVE } \\
(16.9-23.9 \\
20.7) \\
\end{array}$ \\
\hline & Citrobacter sp. & 1 & neg & $\begin{array}{l}\text { POSITIVE } \\
(11.8)\end{array}$ & neg & neg & $\begin{array}{l}\text { POSITIVE } \\
(21.7)\end{array}$ & neg & \begin{tabular}{|l|} 
POSITIVE \\
$(22.9)$
\end{tabular} & neg & neg & neg & \begin{tabular}{|l} 
POSITIVE \\
$(21.0)$
\end{tabular} \\
\hline & Enterobacter cloacae & 10 & neg & $\begin{array}{l}\text { POSITIVE } \\
(8.7-15.3 ; \\
12.7)\end{array}$ & neg & neg & $\begin{array}{l}\text { POSITIVE } \\
(21.4-22.2 ; \\
21.8)\end{array}$ & neg & $\begin{array}{l}\text { POSITIVE } \\
(20.4-24.3 ; \\
22.9) \\
\end{array}$ & neg & neg & neg & $\begin{array}{l}\text { POSITIVE } \\
(16.4-23.0 \\
20.9) \\
\end{array}$ \\
\hline & Escherichia coli & 26 & neg & $\begin{array}{l}\text { POSITIVE } \\
(11.3-18.9 \text {; } \\
14.1)\end{array}$ & neg & neg & $\begin{array}{l}\text { POSITIVE } \\
(19.9-22.3 ; \\
21.7)\end{array}$ & neg & $\begin{array}{l}\text { POSITIVE } \\
(19.8-30.3 ; \\
24.4)\end{array}$ & neg & neg & neg & $\begin{array}{l}\text { POSITIVE } \\
(19.7-25.1 \\
22.4) \\
\end{array}$ \\
\hline & Klebsiella pneumoniae & 6 & neg & $\begin{array}{l}\text { POSITIVE } \\
(12.4-15.2 ; \\
13.8)\end{array}$ & neg & neg & $\begin{array}{l}\text { POSITIVE } \\
(21.7-22.1 ; \\
21.9)\end{array}$ & neg & $\begin{array}{l}\text { POSITIVE } \\
(23.6-25.1 ; \\
24.2)\end{array}$ & neg & neg & neg & $\begin{array}{l}\text { POSITIVE } \\
(21.2-23.3 \\
22.1) \\
\end{array}$ \\
\hline & \multirow{2}{*}{$\begin{array}{l}\text { Pseudocitrobacter } \\
\text { faecalis }\end{array}$} & 1 & neg & $\begin{array}{l}\text { POSITIVE } \\
(13.3)\end{array}$ & neg & neg & $\begin{array}{l}\text { POSITIVE } \\
(21.3)\end{array}$ & neg & \begin{tabular}{|l|} 
POSITIVE \\
$(23.7)$
\end{tabular} & neg & neg & neg & \begin{tabular}{|l} 
POSITIVE \\
$(22.4)$
\end{tabular} \\
\hline & & 1 & neg & $\begin{array}{l}\text { POSITIVE } \\
(13.0)\end{array}$ & $\begin{array}{l}\text { POSITIVE } \\
(27.8)\end{array}$ & neg & $\begin{array}{l}\text { POSITIVE } \\
(21.3)\end{array}$ & neg & \begin{tabular}{|l} 
POSITIVE \\
$(23.3)$
\end{tabular} & neg & neg & neg & \begin{tabular}{|l|} 
POSITIVE \\
$(21.2)$ \\
\end{tabular} \\
\hline & Providencia rettgeri & 1 & neg & $\begin{array}{l}\text { POSITIVE } \\
(9.7)\end{array}$ & neg & neg & $\begin{array}{l}\text { POSITIVE } \\
(21.3)\end{array}$ & neg & \begin{tabular}{|l|} 
POSITIVE \\
$(20.6)$ \\
\end{tabular} & neg & neg & neg & \begin{tabular}{|l} 
POSITIVE \\
(18.4) \\
\end{tabular} \\
\hline \multirow[b]{2}{*}{$\begin{array}{l}\text { OXA-48- } \\
\text { like }\end{array}$} & Escherichia coli & 1 & neg & neg & $\begin{array}{l}\text { POSITIVE } \\
(16.7)\end{array}$ & neg & $\begin{array}{l}\text { POSITIVE } \\
(22.6)\end{array}$ & neg & neg & \begin{tabular}{|l} 
POSITIVE \\
$(25.4)$
\end{tabular} & neg & neg & \begin{tabular}{|l|} 
POSITIVE \\
$(26.2)$
\end{tabular} \\
\hline & Klebsiella pneumoniae & 39 & neg & neg & $\begin{array}{l}\text { POSITIVE } \\
(10.6-15.5 ; \\
13.0)\end{array}$ & neg & $\begin{array}{l}\text { POSITIVE } \\
(21.3-24.8 ; \\
23.0)\end{array}$ & neg & neg & $\begin{array}{l}\text { POSITIVE } \\
(19.1-24.7 ; \\
22.2) \\
\end{array}$ & neg & neg & $\begin{array}{l}\text { POSITIVE } \\
(19.9-28.2 \\
23.0) \\
\end{array}$ \\
\hline \multirow[t]{8}{*}{ IMP-4-like } & Citrobacter freundii & 3 & neg & neg & neg & $\begin{array}{l}\text { POSITIVE } \\
(15.1-16.3 ; \\
15.6)\end{array}$ & $\begin{array}{l}\text { POSITIVE } \\
(20.8-21.9 \text {; } \\
21.3)\end{array}$ & neg & neg & neg & $\begin{array}{l}\text { POSITIVE } \\
(19.7-21.5 ; \\
20.7) \\
\end{array}$ & neg & $\begin{array}{l}\text { POSITIVE } \\
(18.6-21.9 \\
20.6) \\
\end{array}$ \\
\hline & Enterobacter asburiae & 1 & neg & neg & neg & $\begin{array}{l}\text { POSITIVE } \\
(17.0)\end{array}$ & $\begin{array}{l}\text { POSITIVE } \\
(22.0)\end{array}$ & neg & neg & neg & \begin{tabular}{|l|} 
POSITIVE \\
$(21.1)$
\end{tabular} & neg & $\begin{array}{l}\text { POSITIVE } \\
(20.2) \\
\end{array}$ \\
\hline & Enterobacter cloacae & 5 & neg & neg & neg & $\begin{array}{l}\text { POSITIVE } \\
(11.7-18.1 ; \\
16.5)\end{array}$ & $\begin{array}{l}\text { POSITIVE } \\
(20.7-21.9 \text {; } \\
21.4)\end{array}$ & neg & neg & neg & $\begin{array}{l}\text { POSITIVE } \\
(17.4-22.4 ; \\
21.0) \\
\end{array}$ & neg & $\begin{array}{l}\text { POSITIVE } \\
(17.4-21.3 \\
20.1) \\
\end{array}$ \\
\hline & Escherichia coli & 3 & neg & neg & neg & $\begin{array}{l}\text { POSITIVE } \\
\text { (13.5-16.5; } \\
15.4)\end{array}$ & $\begin{array}{l}\text { POSITIVE } \\
(20.7-22.5 ; \\
21.4)\end{array}$ & neg & neg & neg & $\begin{array}{l}\text { POSITIVE } \\
(19.2-22.4 ; \\
21.0) \\
\end{array}$ & neg & \begin{tabular}{|l} 
POSITIVE \\
$(20.3-22.6$ \\
$21.1)$ \\
\end{tabular} \\
\hline & $\begin{array}{l}\text { Enterobacter } \\
\text { hormarchei }\end{array}$ & 1 & neg & neg & neg & $\begin{array}{l}\text { POSITIVE } \\
(11.3)\end{array}$ & $\begin{array}{l}\text { POSITIVE } \\
(21.0)\end{array}$ & neg & neg & neg & $\begin{array}{l}\text { POSITIVE } \\
(17.5)\end{array}$ & neg & \begin{tabular}{|l} 
POSITIVE \\
$(16.5)$
\end{tabular} \\
\hline & Klebsiella oxytoca & 2 & neg & neg & neg & $\begin{array}{l}\text { POSITIVE } \\
(16.0-16.3 ; \\
16.2)\end{array}$ & $\begin{array}{l}\text { POSITIVE } \\
(21.9-22.2 \text {; } \\
22.0)\end{array}$ & neg & neg & neg & $\begin{array}{l}\text { POSITIVE } \\
(20.0-20.2 \text {; } \\
20.1)\end{array}$ & neg & $\begin{array}{l}\text { POSITIVE } \\
(20.4-20.9 \\
20.7) \\
\end{array}$ \\
\hline & Klebsiella pneumoniae & 3 & neg & neg & neg & $\begin{array}{l}\text { POSITIVE } \\
(16.2-17.2 \text {; } \\
16.7)\end{array}$ & $\begin{array}{l}\text { POSITIVE } \\
(21.8-22.2 \text {; } \\
22.1)\end{array}$ & neg & neg & neg & $\begin{array}{l}\text { POSITIVE } \\
(20.2-21.6 ; \\
20.9)\end{array}$ & neg & $\begin{array}{l}\text { POSITIVE } \\
(19.5-21.4 \\
20.6) \\
\end{array}$ \\
\hline & Serratia marcescens & 1 & neg & neg & neg & $\begin{array}{l}\text { POSITIVE } \\
(18.8)\end{array}$ & $\begin{array}{l}\text { POSITIVE } \\
(22.5)\end{array}$ & neg & neg & neg & $\begin{array}{l}\text { POSITIVE } \\
(22.0)\end{array}$ & neg & $\begin{array}{l}\text { POSITIVE } \\
(22.9) \\
\end{array}$ \\
\hline \multirow[t]{2}{*}{ VIM } & \multirow{2}{*}{$\begin{array}{l}\text { Pseudomonas } \\
\text { aeruginosa }\end{array}$} & 21 & neg & neg & neg & $\begin{array}{l}\text { POSITIVE } \\
(12.4-17.2 \text {; } \\
15.0)\end{array}$ & $\begin{array}{l}\text { POSITIVE } \\
(22.7-23.8 ; \\
23.2)\end{array}$ & neg & neg & neg & neg & $\begin{array}{l}\text { POSITIVE } \\
(22.2-27.4 ; \\
24.4)\end{array}$ & $\begin{array}{l}\text { POSITIVE } \\
(20.9-25.0 \\
23.2) \\
\end{array}$ \\
\hline & & 1 & neg & neg & $\begin{array}{l}\text { POSITIVE } \\
(30.9)\end{array}$ & $\begin{array}{l}\text { POSITIVE } \\
(15.8)\end{array}$ & $\begin{array}{l}\text { POSITIVE } \\
(23.4)\end{array}$ & neg & neg & neg & neg & $\begin{array}{l}\text { POSITIVE } \\
(20.2)\end{array}$ & \begin{tabular}{|l}
$\begin{array}{l}\text { POSITIVE } \\
(18.0)\end{array}$ \\
\end{tabular} \\
\hline $\begin{array}{l}\text { NDM + } \\
\text { OXA-48- } \\
\text { like }\end{array}$ & Klebsiella pneumoniae & 6 & neg & $\begin{array}{l}\text { POSITIVE } \\
(11.4-14.1 ; \\
12.8) \\
\end{array}$ & $\begin{array}{l}\text { POSITIVE } \\
(9.7-12.0 \text {; } \\
11.2) \\
\end{array}$ & neg & $\begin{array}{l}\text { POSITIVE } \\
(22.1-23.7 ; \\
22.6)\end{array}$ & neg & $\begin{array}{l}\text { POSITIVE } \\
(20.8-22.8 ; \\
21.8) \\
\end{array}$ & \begin{tabular}{|l} 
POSITIVE \\
$(18.2-21.9 ;$ \\
$20.4)$ \\
\end{tabular} & neg & neg & $\begin{array}{l}\text { POSITIVE } \\
(19.6-23.8 \\
21.8) \\
\end{array}$ \\
\hline $\begin{array}{l}\text { KPC + } \\
\text { NDM } \\
\end{array}$ & Klebsiella pneumoniae & 1 & $\begin{array}{l}\text { POSITIVE } \\
(12.6)\end{array}$ & $\begin{array}{l}\text { POSITIVE } \\
(34.7)\end{array}$ & neg & neg & $\begin{array}{l}\text { POSITIVE } \\
(22.7)\end{array}$ & $\begin{array}{l}\text { POSITIVE } \\
(22.9)\end{array}$ & \begin{tabular}{|l|} 
POSITIVE \\
$(39.5)$
\end{tabular} & neg & neg & neg & \begin{tabular}{|l} 
POSITIVE \\
$(22.4)$ \\
\end{tabular} \\
\hline $\begin{array}{l}\text { OXA-48- } \\
\text { like + VIM }\end{array}$ & Klebsiella pneumoniae & 1 & neg & neg & $\begin{array}{l}\text { POSITIVE } \\
(10.3)\end{array}$ & $\begin{array}{l}\text { POSITIVE } \\
(24.4)\end{array}$ & $\begin{array}{l}\text { POSITIVE } \\
(23.1)\end{array}$ & neg & neg & \begin{tabular}{|l|} 
POSITIVE \\
$(19.3)$
\end{tabular} & neg & $\begin{array}{l}\text { POSITIVE } \\
(36.4)\end{array}$ & $\begin{array}{l}\text { POSITIVE } \\
(20.2)\end{array}$ \\
\hline \multirow{4}{*}{ None } & Enterobacter aerogenes & 5 & neg & neg & neg & neg & $\begin{array}{l}\text { POSITIVE } \\
(21.2-22.0 \text {; } \\
21.5)\end{array}$ & neg & neg & neg & neg & neg & $\begin{array}{l}\text { POSITIVE } \\
(19.5-22.2 \\
21.0) \\
\end{array}$ \\
\hline & Escherichia coli & 6 & neg & neg & neg & neg & $\begin{array}{l}\text { POSITIVE } \\
(21.1-22.0 ; \\
21.5)\end{array}$ & neg & neg & neg & neg & neg & \begin{tabular}{|l} 
POSITIVE \\
$(23.3-26.0$ \\
$24.6)$ \\
\end{tabular} \\
\hline & Klebsiella pneumoniae & 6 & neg & neg & neg & neg & $\begin{array}{l}\text { POSITIVE } \\
(21.3-21.7 ; \\
21.5)\end{array}$ & neg & neg & neg & neg & neg & $\begin{array}{l}\text { POSITIVE } \\
(17.3-21.6 \\
19.2) \\
\end{array}$ \\
\hline & $\begin{array}{l}\text { Pseudomonas } \\
\text { aeruginosa }\end{array}$ & 7 & neg & neg & neg & neg & $\begin{array}{l}\text { POSITIVE } \\
(20.9-21.5 ; \\
21.3)\end{array}$ & neg & neg & neg & neg & neg & \begin{tabular}{|l} 
POSITIVE \\
$(18.4-26.2$ \\
$20.9)$ \\
\end{tabular} \\
\hline
\end{tabular}

neg negative

during which fluorescent signal is not acquired, hence samples tested by Carba (beta) assay typically provided $\mathrm{Ct}$ values that were approximately 10 cycles earlier than in-house PCR methods utilising conventional cycling.
Faecal specimens

Of the 460 faecal DNA extracts tested, 452 were negative for carbapenemase targets by the Carba (beta) assay (see Table 4). Seven samples provided positive results for 
Table 4 Results for faecal swab clinical extracts $(n=460)$. Regarding ' $n / a$ ' results, only clinical extracts positive by the Carba (beta) assay were subjected to in-house assays and DNA sequencing

\begin{tabular}{|c|c|c|c|c|c|c|c|c|c|c|c|c|}
\hline \multirow[t]{2}{*}{$n$} & \multicolumn{5}{|c|}{ Carba (beta) assay Ct values (range; mean) } & \multicolumn{6}{|c|}{ In-house assays Ct values (range; mean) } & \multirow{2}{*}{$\begin{array}{l}\text { Sequencing } \\
\text { result }\end{array}$} \\
\hline & $\overline{\mathrm{KPC}}$ & NDM & OXA-48-like & IMP-4-likeNIM & IC & $\overline{\mathrm{KPC}}$ & NDM & OXA-48-like & IMP-4-like & VIM & $16 \mathrm{~S}$ rRNA & \\
\hline 1 & neg & neg & neg & $\begin{array}{l}\text { POSITIVE } \\
(25.4)\end{array}$ & $\begin{array}{l}\text { POSITIVE } \\
(22.6)\end{array}$ & n/a & $\mathrm{n} / \mathrm{a}$ & $\mathrm{n} / \mathrm{a}$ & $\begin{array}{l}\text { POSITIVE } \\
(31.4)\end{array}$ & neg & $\begin{array}{l}\text { POSITIVE } \\
(11.2)\end{array}$ & IMP-4 \\
\hline 1 & neg & neg & $\begin{array}{l}\text { POSITIVE } \\
(27.0)\end{array}$ & neg & $\begin{array}{l}\text { POSITIVE } \\
(23.4)\end{array}$ & $\mathrm{n} / \mathrm{a}$ & $\mathrm{n} / \mathrm{a}$ & neg & $\mathrm{n} / \mathrm{a}$ & n/a & $\begin{array}{l}\text { POSITIVE } \\
(15.5)\end{array}$ & OXA-181 \\
\hline 6 & neg & neg & $\begin{array}{l}\text { POSITIVE } \\
(25.1-28.9 ; 27.1)\end{array}$ & neg & $\begin{array}{l}\text { POSITIVE } \\
(22.3-24.4 ; 23.0)\end{array}$ & n/a & $\mathrm{n} / \mathrm{a}$ & neg & $\mathrm{n} / \mathrm{a}$ & $\mathrm{n} / \mathrm{a}$ & $\begin{array}{l}\text { POSITIVE } \\
(12.4-18.7 ; 14.2)\end{array}$ & $\mathrm{n} / \mathrm{a}$ \\
\hline 452 & neg & neg & neg & neg & $\begin{array}{l}\text { POSITIVE } \\
(21.4-28.7 ; 22.8)\end{array}$ & $\mathrm{n} / \mathrm{a}$ & $\mathrm{n} / \mathrm{a}$ & $\mathrm{n} / \mathrm{a}$ & $\mathrm{n} / \mathrm{a}$ & $\mathrm{n} / \mathrm{a}$ & $\mathrm{n} / \mathrm{a}$ & $\mathrm{n} / \mathrm{a}$ \\
\hline
\end{tabular}

neg negative

$n / a$ not available

OXA-48-like (Ct values 25.1 to 28.9 cycles), and one sample for IMP-4-like/VIM (25.4 cycles). When tested by the in-house assays, the seven OXA-48-like samples provided negative results, whereas the IMP-4-like/VIM positive sample produced a positive result in the IMP-4like PCR (Ct value of 31.4 cycles). DNA sequencing was attempted for all eight samples; one of the OXA-48-like positive samples returned a sequence confirmed as OXA-181, and the IMP-4-like/VIM positive sample returned an IMP-4 sequence. Amplification for DNA sequencing was not successful for the other samples.

\section{Assay detection limits}

The Carba (beta) assay detection limit was the same for NDM, KPC and VIM targets, and was more sensitive for OXA-48-like and IMP-4-like targets, in comparison to the corresponding in-house assays (see Table 5). The detection limits for the Carba (beta) assay were approximately $1.5 \mathrm{CFU} / \mu \mathrm{L}$ for all five targets. Detection limits for the in-house assays were approximately $15 \mathrm{CFU} / \mu \mathrm{L}$ for IMP-4-like and OXA-48-like assays and 1.5 CFU/ $\mu \mathrm{L}$ for KPC, NDM and VIM assays.

\section{Discussion}

The results indicate the Carba (beta) assay is suitable for detection of carbapenemase genes in cultured isolates. Correct results were provided for 154 of $156 \mathrm{CPO}$ isolates and 24 of 24 non-CPO isolates, giving the Carba (beta) assay $100 \%$ sensitivity and $\geq 98.5 \%$ specificity for cultured isolate extracts. From a practical standpoint, the single reaction format of the Carba (beta) assay offers significant advantages in terms of isolate testing throughput over our current in-house methods, which currently require three reactions per isolate.

The reduced OXA-48-like specificity observed for the Carba (beta) assay was due to two isolates (P. faecalis and P. aeruginosa) that gave OXA-48-like Ct values of 27.8 and 30.9 cycles. These were negative by the OXA-48-like in-house assay and DNA sequencing, and subsequently provided negative results in the Carba (beta) assay when

Table 5 Detection limits for Carba (beta) assay and in-house assays. Performance was assessed individually (i.e. with five isolates possessing only one of the assayed genes) using isolate extracts characterised in this study. All Ct values for replicates at each dilution level are shown. Results beyond the limit of detection for each assay and gene are shaded in grey

\begin{tabular}{|c|c|c|c|c|c|c|c|c|c|c|c|c|}
\hline \multirow[b]{2}{*}{$\begin{array}{l}\text { CFU } / \mu \mathrm{L} \\
\text { of DNA } \\
\text { extract }\end{array}$} & \multicolumn{2}{|c|}{ CFU per reaction ${ }^{*}$} & \multicolumn{2}{|c|}{ KPC Ct values } & \multicolumn{2}{|c|}{ NDM Ct values } & \multicolumn{2}{|c|}{ OXA-48-like Ct values } & \multicolumn{2}{|c|}{ IMP-4-like Ct values } & \multicolumn{2}{|c|}{ VIM Ct values } \\
\hline & $\begin{array}{l}\text { Carba } \\
\text { (beta) } \\
\text { assay }\end{array}$ & $\begin{array}{l}\text { In-house } \\
\text { assay }\end{array}$ & $\begin{array}{l}\text { Carba (beta) } \\
\text { assay }\end{array}$ & $\begin{array}{l}\text { In-house } \\
\text { assay }\end{array}$ & $\begin{array}{l}\text { Carba (beta) } \\
\text { assay }\end{array}$ & $\begin{array}{l}\text { In-house } \\
\text { assay }\end{array}$ & $\begin{array}{l}\text { Carba (beta) } \\
\text { assay }\end{array}$ & $\begin{array}{l}\text { In-house } \\
\text { assay }\end{array}$ & $\begin{array}{l}\text { Carba (beta) } \\
\text { assay }\end{array}$ & $\begin{array}{l}\text { In-house } \\
\text { assay }\end{array}$ & $\begin{array}{l}\text { Carba (beta) } \\
\text { assay }\end{array}$ & $\begin{array}{l}\text { In-house } \\
\text { assay }\end{array}$ \\
\hline 150000 & 750000 & 300000 & $\begin{array}{l}13.5,13.6, \\
13.5\end{array}$ & $\begin{array}{l}19.8,19.8, \\
19.6\end{array}$ & $\begin{array}{l}13.7,13.7, \\
13.6\end{array}$ & $\begin{array}{l}19.5,19.4, \\
19.4\end{array}$ & $\begin{array}{l}12.0,11.9, \\
12.0\end{array}$ & $\begin{array}{l}19.8,20.0, \\
20.1\end{array}$ & $\begin{array}{l}13.3,13.3, \\
13.2\end{array}$ & $\begin{array}{l}18.8,18.7, \\
18.7\end{array}$ & $\begin{array}{l}13.5,13.4, \\
13.4\end{array}$ & $\begin{array}{l}20.2,19.9, \\
20.0\end{array}$ \\
\hline 15000 & 75000 & 30000 & $\begin{array}{l}16.4,16.4, \\
16.6\end{array}$ & $\begin{array}{l}22.1,22.0, \\
21.7\end{array}$ & $\begin{array}{l}16.0,15.9 \\
15.7 \\
\end{array}$ & \begin{tabular}{|l|}
$21.5,21.5$, \\
21.6
\end{tabular} & $\begin{array}{l}14.1,13.8, \\
13.8\end{array}$ & $\begin{array}{l}23.3,23.5, \\
22.6\end{array}$ & $\begin{array}{l}16.6,16.4, \\
16.3\end{array}$ & $\begin{array}{l}21.7,21.5, \\
21.5\end{array}$ & $\begin{array}{l}16.0,15.9, \\
15.8\end{array}$ & $\begin{array}{l}23.0,22.8, \\
22.9\end{array}$ \\
\hline 1500 & 7500 & 3000 & $\begin{array}{l}19.6,19.5, \\
19.4\end{array}$ & $\begin{array}{l}25.3,25.3, \\
25.5\end{array}$ & $\begin{array}{l}18.9,18.6, \\
18.4\end{array}$ & $\begin{array}{l}24.9,25.2 \\
25.2\end{array}$ & $\begin{array}{l}17.4,17.2, \\
17.1\end{array}$ & $\begin{array}{l}26.7,26.2, \\
26.1\end{array}$ & $\begin{array}{l}20.1,19.9, \\
19.5\end{array}$ & $\begin{array}{l}24.9,24.7, \\
24.7\end{array}$ & $\begin{array}{l}18.9,18.8, \\
18.7\end{array}$ & $\begin{array}{l}26.1,26.3, \\
26.5\end{array}$ \\
\hline 150 & 750 & 300 & $\begin{array}{l}23.1,22.9, \\
22.8\end{array}$ & $\begin{array}{l}28.9,28.4, \\
28.8\end{array}$ & \begin{tabular}{|l|}
$21.8,22.1$, \\
21.4
\end{tabular} & \begin{tabular}{|l|}
$28.3,28.5$, \\
28.6 \\
\end{tabular} & $\begin{array}{l}20.6,20.4, \\
20.3\end{array}$ & $\begin{array}{l}30.0,29.8, \\
29.9\end{array}$ & $\begin{array}{l}23.1,23.4, \\
22.9\end{array}$ & $\begin{array}{l}28.4,28.1, \\
28.8\end{array}$ & $\begin{array}{l}22.0,21.7, \\
21.6\end{array}$ & $\begin{array}{l}29.0,28.9, \\
28.5\end{array}$ \\
\hline 15 & 75 & 30 & $\begin{array}{l}25.9,25.4, \\
25.7,26.0, \\
25.8\end{array}$ & $\begin{array}{l}32.8,32.5 \\
32.1,32.9 \\
33.0\end{array}$ & $\begin{array}{l}24.4,24.5, \\
24.5,23.9, \\
24.1\end{array}$ & \begin{tabular}{|l|}
$31.9,31.7$, \\
$31.8,31.8$, \\
31.6
\end{tabular} & $\begin{array}{l}23.3,23.5, \\
23.0,23.1, \\
23.3\end{array}$ & $\begin{array}{l}34.4,33.0, \\
33.9,33.1, \\
35.8\end{array}$ & $\begin{array}{l}26.2,26.7 \\
25.7,25.4 \\
25.3\end{array}$ & $\begin{array}{l}32.0,34.6,32, \\
34.2,36.0\end{array}$ & $\begin{array}{l}24.3,24.5, \\
23.5,24.5, \\
24.6\end{array}$ & $\begin{array}{l}32.9,31.9, \\
33.2,31.9, \\
32.2\end{array}$ \\
\hline 1.5 & 7.5 & 3 & $\begin{array}{l}28.6,28.7, \\
30.4,30.2, \\
29.3\end{array}$ & $\begin{array}{l}35.8,36,37.5 \\
37.7,36.7\end{array}$ & $\begin{array}{l}27.5,26.6, \\
27.1,27.3, \\
27.5\end{array}$ & \begin{tabular}{|l}
$34.5,34.8$, \\
$35.2,35.3$, \\
36.4 \\
\end{tabular} & $\begin{array}{l}25.5,26.2, \\
26.5,26.3, \\
25.1\end{array}$ & $\begin{array}{l}36.8,37.5, \\
\text { neg, } 36.5, \text { neg }\end{array}$ & $\begin{array}{l}31.9,29.3, \\
30.2,30.7, \\
29.4\end{array}$ & $\begin{array}{l}\text { neg, neg, } 40.5 \text {, } \\
\text { neg, neg }\end{array}$ & $\begin{array}{l}27.9,26.2, \\
27.8,26.6, \\
27.2\end{array}$ & $\begin{array}{l}36.9,37.7, \\
36.0,37.5, \\
38.6\end{array}$ \\
\hline 0.15 & 0.75 & 0.3 & $\begin{array}{l}\text { neg, neg, neg, } \\
\text { neg, neg }\end{array}$ & $\begin{array}{l}\text { neg, neg, neg, } \\
\text { neg, } 39.9\end{array}$ & $\begin{array}{l}\text { neg, neg, neg, } \\
\text { neg, neg }\end{array}$ & \begin{tabular}{|l|}
$37.3,39.2$, \\
neg, 38.1, neg \\
\end{tabular} & $\begin{array}{l}\text { neg, neg, neg, } \\
\text { neg, neg }\end{array}$ & $\begin{array}{l}\text { neg, neg, } 38.5 \text {, } \\
\text { neg, neg }\end{array}$ & $\begin{array}{l}\text { neg, neg, neg, } \\
36.8, \text { neg }\end{array}$ & $\begin{array}{l}\text { neg, neg, neg, } \\
\text { neg, neg }\end{array}$ & $\begin{array}{l}29.4, \text { neg, } \\
33.9, \text { neg, } 29.2\end{array}$ & $\begin{array}{l}\text { neg, neg, neg, } \\
40.5, \text { neg }\end{array}$ \\
\hline 0.015 & 0.075 & 0.03 & $\begin{array}{l}\text { neg, neg, neg, } \\
\text { neg, neg }\end{array}$ & $\begin{array}{l}\text { neg, neg, neg, } \\
\text { neg, neg }\end{array}$ & $\begin{array}{l}\text { neg, neg, neg, } \\
\text { neg, neg }\end{array}$ & $\begin{array}{l}\text { neg, neg, neg, } \\
\text { neg, neg }\end{array}$ & $\begin{array}{l}\text { neg, neg, neg, } \\
\text { neg, neg }\end{array}$ & $\begin{array}{l}\text { neg, neg, neg, } \\
\text { neg, neg }\end{array}$ & $\begin{array}{l}\text { neg, neg, neg, } \\
\text { neg, neg }\end{array}$ & $\begin{array}{l}\text { neg, neg, neg, } \\
\text { neg, neg }\end{array}$ & $\begin{array}{l}\text { neg, neg, neg, } \\
\text { neg, neg }\end{array}$ & $\begin{array}{l}\text { neg, neg, neg, } \\
\text { neg, neg }\end{array}$ \\
\hline
\end{tabular}


re-extracted and retested. Negative results following reextraction and retesting were also observed for a further two isolates that initially provided Ct values of 34.7 cycles and 24.4 cycles for NDM and VIM respectively in the Carba (beta) assay. Such high Ct values are indicative of low DNA loads, and likely suggests these isolates were contaminated with other genomic DNA during the original sample preparation. Employing a Ct value cut-off in the Carba (beta) assay may help to address these issues, but additional experimentation would be needed to establish appropriate cut-offs for the isolates and different types of clinical samples.

The initial results from the direct faecal sample testing suggest the Carba (beta) assay may be suitable for screening purposes, but more work is needed here. The majority of samples (98.3\%; 452/460) returned negative results. This low detection rate is consistent with previous studies indicating these genes are at low prevalence in our local Australian population [21]. However, of the eight positive samples by the Carba (beta) assay (seven for OXA-48-like and one for IMP-4-like/VIM), only two of these positives were confirmed by in-house PCR and/or DNA sequencing (one IMP-4 and one OXA-181). It is possible that OXA-48-like genes may be present in the six non-confirmed samples, and were only detected by the Carba (beta) assay due to its more sensitive detection limit; as seen in Tables 4 and 5 , the OXA-48-like $\mathrm{Ct}$ values provided by the faecal samples were similar to the $\mathrm{Ct}$ value for the limit of OXA-48-like detection. Alternatively, these may be due to contamination as discussed earlier. We were unable to investigate this further as, unlike the isolates, the original faecal samples were not available for re-extraction and retesting. Nevertheless, further validation of direct sample testing is warranted, including from patients at higher risk of harbouring CPOs. In this context, a limitation of this investigation was that we only tested a bank of faecal samples from patients with suspected gastrointestinal infection from a low-CPO prevalence setting. An additional limitation of this study was that we did not assess the detection limit of the assays for each of the individual gene variants.

\section{Conclusion}

In summary, the Carba (beta) assay developed by SpeeDx was sensitive and specific for detecting carbapenemase genes in isolates. Applying a Ct value cut-off when testing isolate extracts may be useful to enhance performance. Further studies are required to determine the suitability of this assay for direct testing of clinical samples.

\section{Abbreviations}

AGRF: Australian Genomic Research Facility; CFU: Colony Forming Unit; CPO: carbapenemase-producing organism; Ct: Cycle threshold; IC: internal controll; IMP: imipenemase; KPC: Klebsiella pneumoniae carbapenemase;
NDM: New Delhi metallo- $\beta$-lactamase; OXA: oxacillin-hydrolyzing; rRNA: ribosomal ribonucleic acid; VIM: Verona integron-encoded metallo- $\beta$ lactamase

\section{Acknowledgements \\ We thank Dr. Sameera Al Johani from King Abdualaziz Medical City in Riyadh, Saudi Arabia, Emad Ibrahim from Hamad Medical Corporation in Qatar and Jameela Alsalman from Salmaniya Medical Complex in Bahrain for providing some of the isolates in this study.}

\section{Authors' contributions}

DMW, RW, HB, HMZ, HES, GRN, DLP and PNAH were involved in study conception and design. Sample extraction and testing was performed by $A B$, $E T, C B, M S$ and KC. Data analysis for the Carba (beta) assay was performed independently by 1) $E T$ and $A B$ from the University of Queensland and 2) MW, RW and LYT from SpeeDx Pty Ltd. Analysis of in-house assay and sequencing data was performed by $E T$ and $A B$. DMW, AB, ET and LYT were major contributors in writing the manuscript. All authors read and approved the final manuscript.

\section{Funding}

This study was funded by SpeeDx Pty Ltd. SpeeDx Pty Ltd. provided the Carba (beta) kits and funding for the consumables used in this study. Co-authors from SpeeDx Pty Ltd. (MW, RW and LYT) participated in study conception and design, data analysis, and manuscript writing as per the 'Authors' contributions' section.

\section{Availability of data and materials}

The datasets for this study are available from the corresponding author on reasonable request. Materials for this study are retained at the University of Queensland Centre for Clinical Research, Australia.

\section{Ethics approval and consent to participate}

The study was approved by the Human Research Ethics Committees (HRECs) of Children's Health Queensland and The University of Queensland (HREC/ 13/QRCH/39). Informed consent was waived as per section 2.3.6 of the NHMRC national statement.

\section{Consent for publication}

Not applicable.

\section{Competing interests}

This study was funded by SpeeDx Pty Ltd. MW, RW and LYT are employees of, and own stock or options in, SpeeDx Pty Ltd. DMW is a member of the editorial board of BMC Infectious Diseases.

\section{Author details}

${ }^{1}$ The University of Queensland, UQ Centre for Clinical Research, Brisbane, Queensland, Australia. ${ }^{2}$ SpeeDx Pty. Ltd., Brisbane, Queensland, Australia. ${ }^{3}$ Pathology Queensland Central Laboratory, Brisbane, Queensland, Australia. ${ }^{4}$ College of Medicine, King Saud bin Abdulaziz University for Health Sciences, Riyadh, Saudi Arabia. ${ }^{5}$ World Health Organization Collaborating Centre for Infection Prevention and Control, Riyadh, Saudi Arabia. ${ }^{6}$ Gulf Cooperation Council Center for Infection Control, Riyadh, Saudi Arabia. ${ }^{7}$ King Abdullah International Medical Research Centre, Riyadh, Saudi Arabia. ${ }^{8}$ Griffith University School of Medicine, Brisbane, Queensland, Australia.

Received: 2 May 2018 Accepted: 10 June 2019

Published online: 02 July 2019

\section{References}

1. Centers for Disease Control and Prevention: Antibiotic Resistance Threats in the United States. 2013; https://www.cdc.gov/drugresistance/threat-report2013/index.html

2. World Health Organisation: Antimicrobial resistance: global report on surveillance. 2014; http://www.who.int/drugresistance/documents/ surveillancereport/en/

3. Tangden T, Giske CG. Global dissemination of extensively drug-resistant carbapenemase-producing Enterobacteriaceae: clinical perspectives on detection, treatment and infection control. J Intern Med. 2015;277:501-12. 
4. Falagas ME, Tansarli GS, Karageorgopoulos DE, Vardakas KZ. Deaths attributable to carbapenem-resistant Enterobacteriaceae infections. Emerg Infect Dis. 2014;20:1170-5.

5. van Duin D, Doi Y. The global epidemiology of carbapenemase-producing Enterobacteriaceae. Virulence. 2017;8:460-9.

6. Nordmann P, Gniadkowski M, Giske CG, Poirel L, Woodford N, Miriagou $V$, European network on Carbapenemases. Identification and screening of carbapenemase-producing Enterobacteriaceae. Clin Microbiol Infect. 2012;18:432-8.

7. Healthcare-associated Infections (HAl): Tracking CRE [https://www.cdc.gov/ hai/organisms/cre/trackingcre.html] Accessed 28 Nov 2017.

8. Tato M, Ruiz-Garbajosa P, Traczewski M, Dodgson A, McEwan A, Humphries R, Hindler J, Veltman J, Wang H, Cantón R. Multisite evaluation of Cepheid Xpert Carba-R assay for detection of Carbapenemase-producing organisms in rectal swabs. J Clin Microbiol. 2016;54:1814-9.

9. Berry C, Davies K, Woodford N, Wilcox M, Chilton C. Survey of screening methods, rates and policies for the detection of carbapenemase producing Enterobacteriaceae in English hospitals. J Hosp Infect. 2018;101(2):158-62.

10. Zowawi HM, Sartor AL, Balkhy HH, Walsh TR, Al Johani SM, AlJindan RY, Alfaresi M, Ibrahim E, Al-Jardani A, Al-Abri S, et al. Molecular characterization of carbapenemase-producing Escherichia coli and Klebsiella pneumoniae in the countries of the Gulf cooperation council: dominance of OXA-48 and NDM producers. Antimicrob Agents Chemother. 2014;58:3085-90.

11. Zarakolu P, Day KM, Sidjabat HE, Kamolvit W, Lanyon CV, Cummings SP, Paterson DL, Akova M, Perry JD. Evaluation of a new chromogenic medium, chromID OXA-48, for recovery of carbapenemase-producing Enterobacteriaceae from patients at a university hospital in Turkey. Eur I Clin Microbiol Infect Dis. 2015;34:519-25.

12. Sidjabat HE, Townell N, Nimmo GR, George NM, Robson J, Vohra R, Davis L, Heney C, Paterson DL. Dominance of IMP-4-producing enterobacter cloacae among carbapenemase-producing Enterobacteriaceae in Australia. Antimicrob Agents Chemother. 2015;59:4059-66.

13. Wailan AM, Sartor AL, Zowawi HM, Perry JD, Paterson DL, Sidjabat HE. Genetic contexts of blaNDM-1 in patients carrying multiple NDM-producing strains. Antimicrob Agents Chemother. 2015:59:7405-10.

14. Doi Y, Potoski BA, Adams-Haduch JM, Sidjabat HE, Pasculle AW, Paterson DL. Simple disk-based method for detection of Klebsiella pneumoniae Carbapenemase-type $\beta$-lactamase by use of a Boronic acid compound. J Clin Microbiol. 2008:46:4083-6.

15. Mokany E, Bone SM, Young PE, Doan TB, Todd AV. MNAzymes, a versatile new class of nucleic acid enzymes that can function as biosensors and molecular switches. J Am Chem Soc. 2010;132:1051-9.

16. Mokany E, Tan YL, Bone SM, Fuery CJ, Todd AV. MNAzyme qPCR with superior multiplexing capacity. Clin Chem. 2013;59:419-26.

17. Mokany E, Todd AV. MNAzyme qPCR: a superior tool for multiplex QPCR. Methods Mol Biol. 2013;1039:31-49.

18. Centers for Disease Control and Prevention: Multiplex real-time PCR detection of Klebsiella pneumoniae Carbapenemase (KPC) and New Delhi metallo- $\beta$-lactamase (NDM-1) genes. Healthcare-associated Infections Laboratory Resources 2011.

19. Naas T, Cotellon G, Ergani A, Nordmann P. Real-time PCR for detection of blaOXA-48 genes from stools. J Antimicrob Chemother. 2013;68:101-4.

20. Carter I. Metallo $\beta$ lactamases gene blaimp, blaspm and blavim detection by multiplex real-time TagMan assay on the Smartcycler. In: Schuller M, Sloots TP, James GS, Halliday CL, Carter IWJ, editors. PCR for clinical microbiology: an Australian and international perspective. Dordrecht: Springer Netherlands; 2010. p. 423-7.

21. Australian Commission on Safety and Quality in Health Care: CARAlert annual report 2016-2017 2017; https:/www.safetyandquality.gov.au/wpcontent/uploads/2017/07/CARAlert-Report-March-2016-to-March-2017.pdf.

\section{Publisher's Note}

Springer Nature remains neutral with regard to jurisdictional claims in published maps and institutional affiliations.

\section{Ready to submit your research? Choose BMC and benefit from:}

- fast, convenient online submission

- thorough peer review by experienced researchers in your field

- rapid publication on acceptance

- support for research data, including large and complex data types

- gold Open Access which fosters wider collaboration and increased citations

- maximum visibility for your research: over $100 \mathrm{M}$ website views per year

At BMC, research is always in progress.

Learn more biomedcentral.com/submissions 